\title{
Radiation Damage in Silicon Photonic Mach-Zehnder Modulators and Photodiodes
}

\author{
Marcel Zeiler ${ }^{\circledR}$, Sarah Seif El Nasr-Storey, Stephane Detraz, Andrea Kraxner, Lauri Olantera, \\ Carmelo Scarcella, Christophe Sigaud, Csaba Soos, Jan Troska, and Francois Vasey
}

\begin{abstract}
Radiation-hard optical links are the backbone of read-out systems in high-energy physics (HEP) experiments at CERN. The optical components must withstand large doses of radiation and strong magnetic fields and provide high data rates. Radiation hardness is one of the requirements that become more demanding with every new generation of HEP experiment. Previous studies have shown that vertical cavity surface emitting lasers, on which the current optical links are based, will not be able to withstand the expected radiation levels in the innermost regions of future HEP experiments. Silicon photonics (SiPh) is currently being investigated as a promising alternative technology to address this challenge. We irradiated SiPh Mach-Zehnder modulators (MZMs) with different design parameters to evaluate their resistance against ionizing radiation. We confirm that $\mathrm{SiPh}$ MZMs with a conventional design do not show a phase shift degradation when exposed to a $20-\mathrm{MeV}$ neutron fluence of $3 \cdot 10^{16} \mathrm{n} / \mathrm{cm}^{2}$. We further demonstrate that custom-designed MZMs with shallow etch optical waveguides and high doping concentrations in their p-n junctions exhibit a strongly improved radiation hardness over devices with a conventional design when irradiated with X-rays. We also found that MZMs withstood higher radiation levels when they were irradiated at a low temperature. In contrast, larger reverse biases during irradiation led to a faster device degradation. Simulations indicate that a pinch-off of holes is responsible for the device degradation. Photodiodes (PDs) were also tested for their radiation hardness as they are needed in silicon photonic transceivers. X-ray irradiation of building-block germanium-silicon PDs showed that they were not significantly affected.
\end{abstract}

Index Terms-CERN, high-energy physics (HEP), ionizing radiation, Large Hadron Collider (LHC), Mach-Zehnder modulator (MZM), neutron radiation, photodiode (PD), radiation damage, silicon photonics (SiPh), X-ray.

Manuscript received May 18, 2017; revised September 4, 2017; accepted September 11, 2017. Date of publication September 20, 2017; date of current version November 14, 2017. This work was supported in part by the Science Foundation Ireland through the IPIC research programme under Grant 12/RC/2276 and in part by the ICE-DIP programme, a European Industrial Doctorate project funded by the European Commission's 7th Framework programme Marie Curie Actions under Grant PITN-GA-2012316596. (Corresponding author: Marcel Zeiler.)

M. Zeiler was with the Experimental Physics Department, CERN, 1211 Geneva, Switzerland. He is now with the Faculty of Engineering and Computing, School of Electronic Engineering, Dublin City University, 9 Dublin, Ireland (e-mail: marcel.zeiler@cern.ch).

S. S. El Nasr-Storey was with the Experimental Physics Department, CERN, 1211 Geneva, Switzerland. She is now with the Faculty of Science, School of Physics, University of Bristol, Bristol BS8 1TH, U.K.

S. Detraz, A. Kraxner, L. Olantera, C. Scarcella, C. Sigaud, C. Soos, J. Troska, and F. Vasey are with the Experimental Physics Department, CERN, 1211 Geneva, Switzerland.

Color versions of one or more of the figures in this paper are available online at http://ieeexplore.ieee.org.

Digital Object Identifier 10.1109/TNS.2017.2754948

\section{INTRODUCTION}

$\mathbf{T}$ HE currently ongoing growth in data traffic is not only limited to Internet applications but also affects large-scale physics experiments. For instance, the data that were sent to CERN's tape archive in the first nine months after the Large Hadron Collider (LHC) restarted for Run 2 were more than 33 PB. In comparison, the data stored during the entire Run 1 (33 months) were roughly 77 PB [1]. This increase in data traffic is predominantly due to proton beams with a higher luminosity and energy. Thereby, more particle collisions are now provided to the high-energy physics (HEP) experiments that in turn create more measurement data. The next upgrade for the LHC is foreseen for the years 2024-2026. The machine will then be upgraded to the high luminosity (HL)-LHC that will have a luminosity five to seven times higher than it is now. This will increase the bandwidth required to send the data to the processing electronics. Moreover, the radiation levels in the innermost detector regions will also be significantly higher than nowadays. Components to be installed in the innermost detector regions of the HL-LHC or future accelerators like the future circular collider will have to withstand a minimum $1 \mathrm{MeV}$-equivalent neutron fluence of more than $6 \cdot 10^{15} \mathrm{n} / \mathrm{cm}^{2}$ and total ionizing dose (TID) levels above $1 \mathrm{MGy}$ (the reference material for TID is $\mathrm{SiO}_{2}$ throughout this paper).

To cope with the expected increase in data traffic and radiation levels, new high-speed transceivers (TRx) are required for the optical links that connect the particle sensors in the radiation zone with the back-end electronics located in the radiation-free counting room. Currently, these TRx are based on vertical cavity surface emitting lasers (VCSELs) and pinphotodiodes (PDs) that have been qualified for operation in the harsh radiation environment. The qualification work has shown, however, that the threshold current of VCSELs increases while their slope efficiency decreases when they are exposed to a neutron fluence as high as expected for the HL-LHC [2]. Beyond HL-LHC, the resulting performance degradation will be too much to be compensated for by the lasers' driving electronics.

We have started investigating silicon photonics $(\mathrm{SiPh})$ as potential technology to replace VCSEL-based radiation-hard optical links for the upstream paths from within the innermost detector regions of future HEP experiments. SiPh could allow the integration of optoelectronic devices with their electronic driving circuits and potentially even with the silicon 
particle sensors. This could lead to a highly integrated readout module with reduced dimensions and mass. Furthermore, the possibility to decouple the feed laser from the modulator in a SiPh-based TRx would allow placing the feed laser a few meters outside the innermost detector regions and connect it to the modulator with an optical fiber. In this way, radiation damage to the laser could be reduced, which would not be possible with VCSELs. In addition, a resistance against radiation similar to that of silicon particle sensors [3], [4] could be expected for $\mathrm{SiPh}$ components. With the components being smaller, lighter, and more resistant against radiation, they could be placed closer to the particle collision points. This could open up the door to higher resolution physics measurements.

We have chosen to investigate a SiPh Mach-Zehnder Modulator (MZM) as modulator for the TRx. An MZM is an interferometric modulator that splits light equally into two separate arms that both contain a phase shifter [see Fig. 3(a)]. Amplitude modulation at the MZM's output can then be achieved by introducing a phase shift between the two arms, constructive interference for zero phase shift and destructive interference for a phase shift of $\pi$. A first neutron irradiation test of SiPh MZMs based on a conventional design (devices as presented in [5]) has indeed shown very promising results. The optical phase shift of these MZMs was not significantly affected when exposed to a $20-\mathrm{MeV}$ neutron fluence of approximately $2 \cdot 10^{15} \mathrm{n} / \mathrm{cm}^{2}$ [6]. We carried out a second neutron irradiation test with the exact same experimental setup as for the first neutron irradiation test. This time, the MZMs with the conventional design were irradiated with a neutron fluence up to $3 \cdot 10^{16} \mathrm{n} / \mathrm{cm}^{2}$. This second test confirmed that they did not show a change in the measured phase shift either (Fig. 1). We attributed the insensitivity of SiPh MZMs to such high neutron fluences to the high doping concentrations used in these devices. A neutron fluence of $1 \cdot 10^{15} \mathrm{n} / \mathrm{cm}^{2}$ creates a defect density in silicon of about $1 \cdot 10^{15} \mathrm{~cm}^{-3}$ [7]. According to the non-ionizing energy loss hypothesis, this relationship should scale linearly to higher neutron fluences. Consequently, a defect density in the order of $1 \cdot 10^{16} \mathrm{~cm}^{-3}$ for a neutron fluence of about $1 \cdot 10^{16} \mathrm{n} / \mathrm{cm}^{2}$ would be one to two orders of magnitude lower than the typical doping concentrations in SiPh MZMs. Hence, neutron-induced defects do not significantly affect the highly doped phase shifters in depletion-type SiPh MZMs.

When exposed to X-rays, however, the phase shift of these MZMs fell to below $20 \%$ of their preirradiation value well before TID levels as expected in the HL-LHC were reached [6]. This degradation was attributed to a pinch-off of holes in the waveguide rib from the electrical contact caused by radiation-induced defects in $\mathrm{SiO}_{2}$. These defects made it impossible to change the free carrier concentrations in the phase shifter and thus create a phase shift [8]. SiPh MZMs with such a conventional design could not be installed in the innermost detector regions of future HEP experiments due to their high sensitivity to ionizing radiation. Previously, we presented a custom-designed SiPh test chip that included several MZMs with varied design parameters [9] to assess whether the resistance against ionizing radiation can be improved through

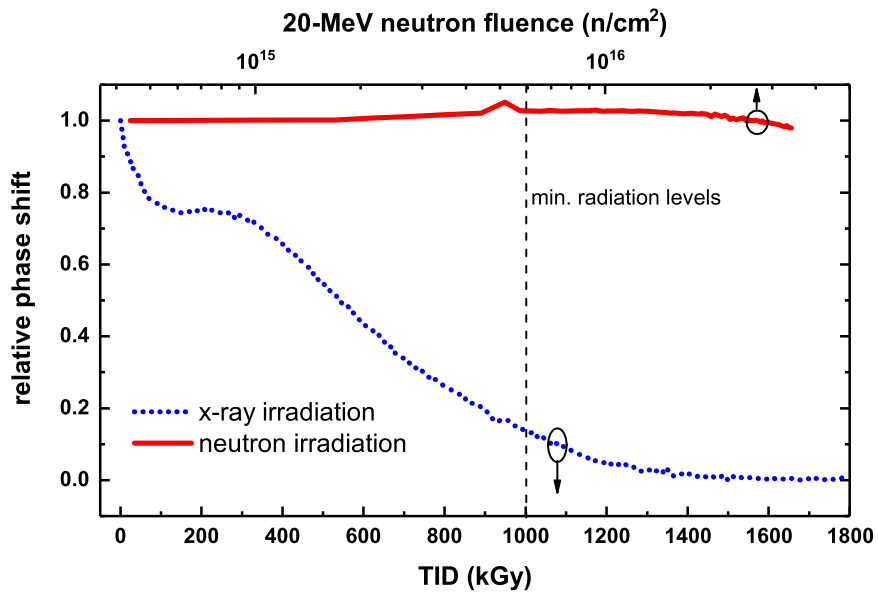

Fig. 1. Change in phase shift (measured at $-3 \mathrm{~V}$ ) of a $\mathrm{SiPh}$ MZM based on a conventional design [5]. The data for the X-ray irradiation were taken from [6].

a "hardening-by-design" approach. In this paper, we present results from three X-ray irradiation campaigns performed on these MZMs.

As the performance of III-V-based PDs degrades similarly to those of VCSELs when exposed to a high neutron fluence [2], we also report on results from an irradiation test of germanium-silicon (GeSi) PDs for use in radiation-hard TRx. In addition to being used as receivers in SiPh-based TRx, GeSi PDs are required as monitor PDs in control loops for MZMs to ensure a stable operation around the MZM's quadrature point [10].

The measurement procedures and test conditions are outlined in Section II. The experimental results are shown in Section III and discussed in Section IV. The conclusion is drawn in Section V.

\section{Test Samples and Measurement Procedures}

\section{A. Devices}

The SiPh MZMs used for all tests were designed by us and fabricated by imec [11] as part of a multiproject wafer (MPW) run offered through ePIXfab [12]. The detailed design parameters of the MZMs are described in [9]. The investigated GeSi PDs were building-block devices designed by imec. They were put on the same mask layout as the MZMs. A cross-sectional view through an MZM's phase shifter showing the design parameters of the investigated devices is depicted in Fig. 2. All MZMs had a waveguide of $450 \mathrm{~nm}$ in width and $220 \mathrm{~nm}$ in height. The length of the phase shifters was $1.9 \mathrm{~mm}$. The two etch depths investigated were either 160 (so-called "deep etch") or $70 \mathrm{~nm}$ (so-called "shallow etch"). The precise p- and $\mathrm{n}$-doping concentrations were not disclosed within the scope of the MPW but are expected to be in the order of $10^{18} \mathrm{~cm}^{-3}$ for MZMs with nominal doping concentration and twice that for devices with $2 \times$ the nominal doping concentration. The $p-n$ junction itself was centered in the waveguide. Contact $(\mathrm{n}++, \mathrm{p}++)$ and intermediate $(\mathrm{n}+, \mathrm{p}+)$ doping concentrations in silicon were not varied. The distance of the intermediate doping levels to the edges of the waveguide ( $\left.w_{\text {Dop }}\right)$ was also varied. MZMs with $w_{\text {Dop }}=500 \mathrm{~nm}$ and $750 \mathrm{~nm}$ were tested. 


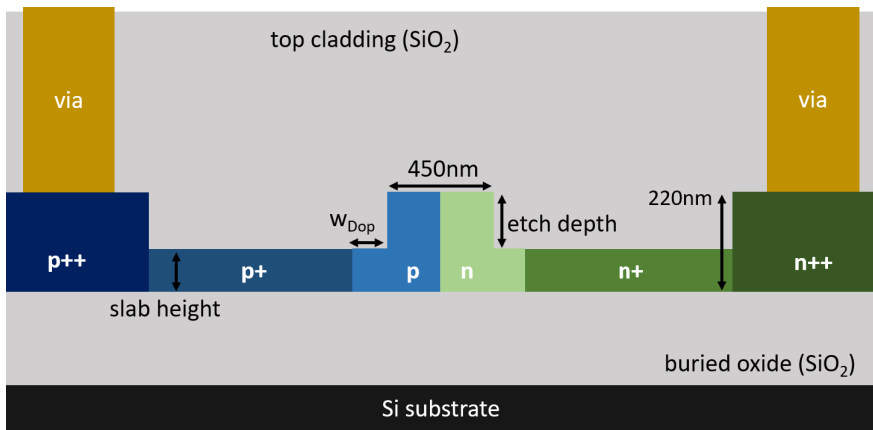

Fig. 2. Cross section through a phase shifter of the investigated MZMs (not to scale). The etch depths and the p- and n-doping concentrations were varied for this paper.

\section{B. Measurement Procedures}

Three X-ray irradiation tests were performed in total. The first test was performed with bare dies that were irradiated stepwise at room temperature and characterized in between the irradiation steps. The devices were not biased while the $\mathrm{X}$-ray source was ON. The second test was also done at room temperature but with pigtailed devices, allowing for biasing during irradiation and in situ measurements. The third test was again a stepwise irradiation of bare dies but at $-30{ }^{\circ} \mathrm{C}$. The measurement procedures and details about the different irradiation tests are outlined below.

1) Phase Shift Measurement: The MZMs' phase shifts were determined by coupling light from a superluminescent LED via a grating coupler into and out of the SiPh chip and recording the transmission spectra as a function of bias voltage $V$ with a Yokogawa AQ6370B optical spectrum analyzer. The test voltages were either applied with probe needles to the MZM on bare dies or through a connector for bonded samples. The phase shift $\Delta \phi$ in $(\pi / \mathrm{mm})$ of a device with length $L$ was calculated based on the wavelength shift $\Delta \lambda$ of the transmission spectra and the free spectral range FSR according to

$$
\Delta \phi=\frac{2 \Delta \lambda}{\mathrm{FSR} \cdot L} .
$$

$\Delta \lambda$ and the FSR were determined from a fit of the recorded spectra. A schematic of the measurement setup and example spectra including fits are shown in Fig. 3.

2) Stepwise Irradiation With Manual Testing: For the first irradiation tests, bare dies were placed in a Seifert RP149 $\mathrm{X}$-ray irradiator and sequentially irradiated to increasing TID levels. The peak energy of the X-rays was $10 \mathrm{keV}$. The dose rate during the test was kept constant to $14.05 \mathrm{~Gy} / \mathrm{s}$. The chips were not biased during irradiation. The phase shifts of the individual MZMs on the dies were manually measured on a probe station before irradiation and after each irradiation step. The chips were exposed to a higher TID directly after the phase shift measurements were finished to minimize annealing. The test was either stopped once a phase shift could no longer be detected or when the irradiation time was up.

The irradiation as well as the measurements in between were all done at room temperature for the first test. One die with nominal doping concentration and one die with $2 \times$ nominal doping concentration were tested separately.

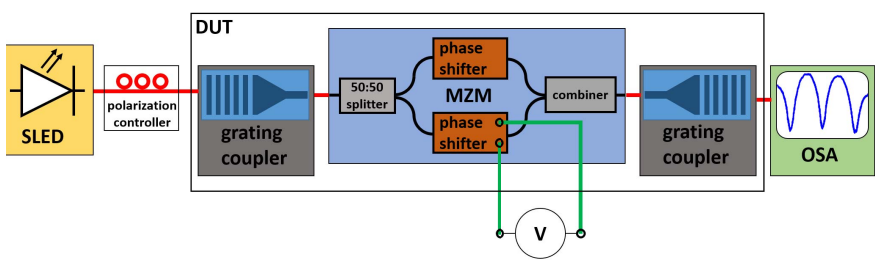

(a)

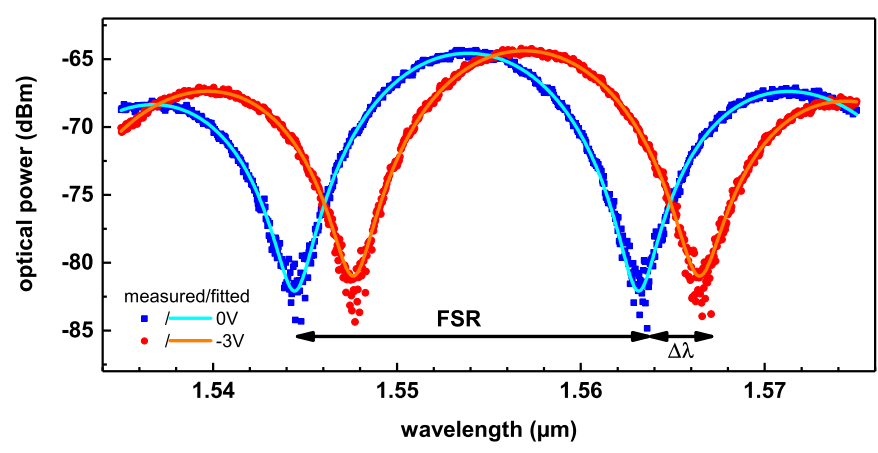

(b)

Fig. 3. (a) Schematic of the measurement setup. (b) Example transmission spectra of a reverse-biased MZM at two voltages.

For the third irradiation test, the temperature during irradiation was lowered to $-30{ }^{\circ} \mathrm{C}$. This would be a similar temperature as found inside in the innermost regions of an actual HEP experiment. Before the temperature could be lowered, wet air had to be removed from the inside of the irradiator to minimize the buildup of ice during the irradiation. The chuck on which the dies were placed was therefore first heated up to $80{ }^{\circ} \mathrm{C}$ for half an hour and the irradiator was at the same time filled with dry air before the temperature was lowered.

If we had used a single die throughout the irradiation test at $-30{ }^{\circ} \mathrm{C}$, heating the test samples before cool down could have caused annealing of radiation-induced defects introduced during the preceding irradiation step. We thus decided to use a new die for each TID step to keep the test conditions as consistent as possible, i.e., one die was irradiated from 0 to $200 \mathrm{kGy}$, another die from 0 to $300 \mathrm{kGy}$, and so on. The preirradiation phase shift values of the tested dies showed a very good uniformity with phase shift values between dies varying less than $10 \%$. Hence, the obtained results can be directly compared with the tests done at room temperature.

3) Continuous Irradiation With Automated Testing: In an actual system installation, MZMs would be biased and modulated during operation. Therefore, pigtailed MZMs were biased and measured during the second irradiation test to evaluate them in a configuration that more closely resembles an actual system installation. The test was done at room temperature because it was not possible to bring the pigtailed samples in contact with the temperature-controlled chuck. The temperature inside the irradiator was monitored during the test and was measured to be $25 \pm 1{ }^{\circ} \mathrm{C}$. Temperature variations affecting the measured performance can thus be excluded. As it was a continuous irradiation, there was no annealing between successive TID steps. 
Aside from minimal annealing that could have occurred when the MZMs were being measured during the stepwise irradiation test, we do not expect that the measurement procedure itself (stepwise or continuous) affected the test results since the test conditions were otherwise identical.

For the automated measurements, dies were glued onto a printed circuit board (PCB) and all MZMs were bonded to it. The test voltage was applied through an electrical connector attached to the PCB. Two planar fiber arrays were aligned to an array of on-die grating couplers to connect optically to the MZMs. Optical and electrical switches were used to successively measure the phase shift of the different MZMs on a die while all the other MZMs were biased at a constant voltage. One die with nominal doping and one die with $2 \times$ nominal doping were tested separately. Both were biased at $-3 \mathrm{~V}$ during irradiation. A third die with $2 \times$ nominal doping was biased at $-1.5 \mathrm{~V}$ during irradiation. The dose rate was the same as for the stepwise irradiation tests.

The dark- and photo-currents of the building-block PDs were measured during the irradiation of the third die. Both currents were measured with a voltage of $-1 \mathrm{~V}$ applied to the PDs. The PDs were biased at the same voltage during irradiation. To measure the photo-current, light from a $1550-\mathrm{nm}$ laser was coupled through the planar fiber arrays onto the PDs. The laser was switched OFF when no photo-current was measured. The photo-currents were not converted to a responsivity as the actual optical power impinging on the PDs was unknown.

\section{EXPERIMENTAL RESULTS}

\section{A. Stepwise Irradiation of MZMs at Room Temperature}

The results obtained from the first stepwise irradiation test with unbiased samples clearly indicate that shallow etch MZMs have a significantly higher radiation hardness than deep etch MZMs (Fig. 4). Additionally, a higher doping concentration also improves the radiation hardness for shallow etch MZMs.

Shallow etch MZMs with nominal doping showed a $17 \%$ increase in phase shift up to 1.7 MGy before it then steeply degraded. However, the phase shift did not drop below their preirradiation value until 1.96 MGy and below $67 \%$ until 2.66 MGy. Shallow etch MZMs with $2 \times$ nominal doping did not show any degradation up to $3.1 \mathrm{MGy}$. Instead, their phase shift increased by $27 \%$. In contrast and independently of the doping concentrations used, deep etch MZMs withstood only a TID level of approximately $100 \mathrm{kGy}$ before their phase shift rapidly dropped to zero around $200 \mathrm{kGy}$. A moderate phase shift enhancement of 6\%-9\% could also be observed for these devices. In all cases, the waveguide doping width $w_{\text {Dop }}$ did not affect the measured phase shift degradation.

\section{B. Continuous Irradiation of MZMs at Room Temperature}

Like for the stepwise irradiation test, shallow etch MZMs with a high doping concentration resisted longer against ionizing radiation than samples with a deeper etch or lower doping when biased at $-3 \mathrm{~V}$ during irradiation (Fig. 5). The phase shift of deep etch MZMs rose by 6\%-15\% before it fell below $50 \%$ of their preirradiation value at a TID level of

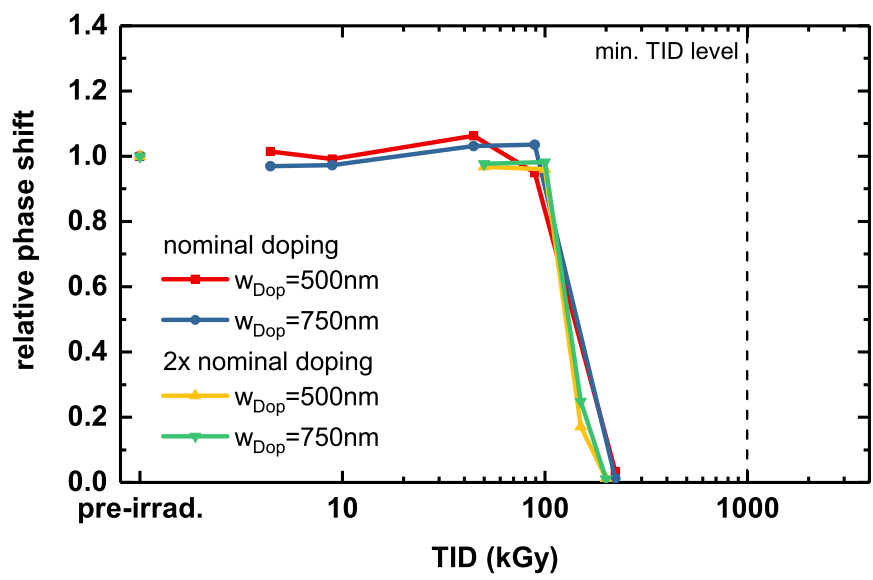

(a)

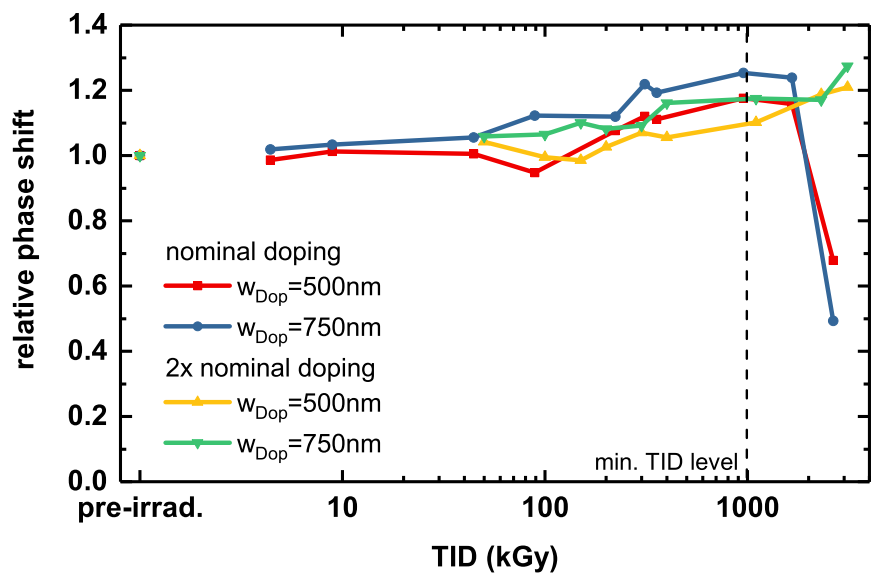

(b)

Fig. 4. Relative phase shift change at $-2 \mathrm{~V}$ as a function of TID obtained during the stepwise irradiation test at room temperature where the MZMs were not biased nor measured during irradiation. (a) Deep etch. (b) Shallow etch.

43 and $75 \mathrm{kGy}$ for devices with nominal and $2 \times$ nominal doping, respectively. Deep etch MZMs no longer showed a phase shift after about $105 \mathrm{kGy}$. A phase shift enhancement of approximately $30 \%$ was measured for shallow etch MZMs with nominal doping. Their phase shift then decreased to 50\% of their preirradiation value at $738 \mathrm{kGy}\left(w_{\text {Dop }}=500 \mathrm{~nm}\right)$ and at $914 \mathrm{kGy}\left(w_{\text {Dop }}=750 \mathrm{~nm}\right)$. The phase shift completely vanished after $1250 \mathrm{kGy}\left(w_{\text {Dop }}=500 \mathrm{~nm}\right)$ and roughly $1560 \mathrm{kGy}\left(w_{\text {Dop }}=750 \mathrm{~nm}\right)$. Shallow etch MZMs with $2 \times$ nominal doping showed a 3\%-9\% increase in phase shift before it dropped below 50\% at $1.3 \mathrm{MGy}$. The phase shift for MZMs with $w_{\text {Dop }}=750 \mathrm{~nm}$ completely disappeared at $1800 \mathrm{kGy}$, while there was still a small phase shift left at $2353 \mathrm{kGy}$ for shallow etch MZMs with $w_{\text {Dop }}=500 \mathrm{~nm}$. Like for the stepwise irradiation test, there was no clear tendency as to whether larger or smaller values for the waveguide doping width $w_{\text {Dop }}$ lead to a better radiation hardness.

The phase shift degradations of shallow etch MZMs with $2 \times$ nominal doping under different bias conditions are compared in Fig. 6. For a reverse bias of $-1.5 \mathrm{~V}$ during irradiation, the phase shift of shallow etch MZMs did not fall below 50\% 


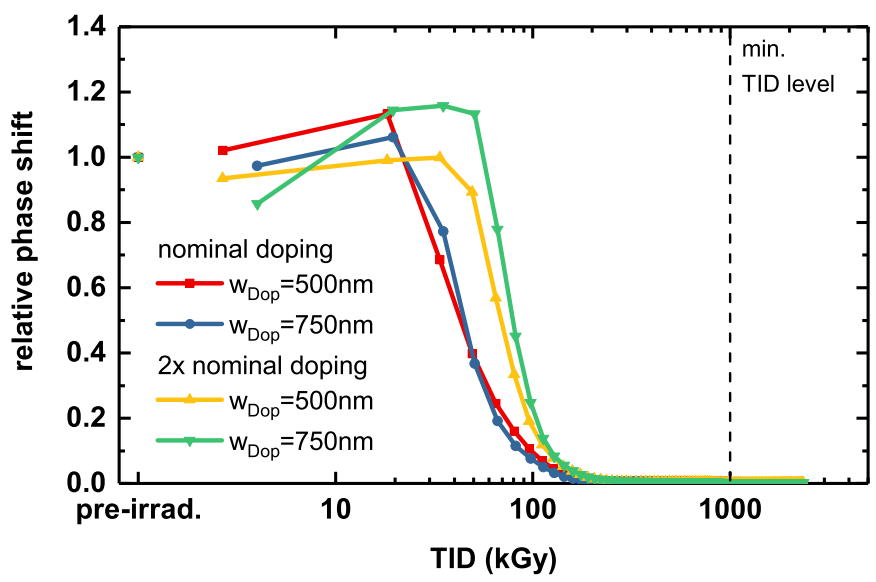

(a)

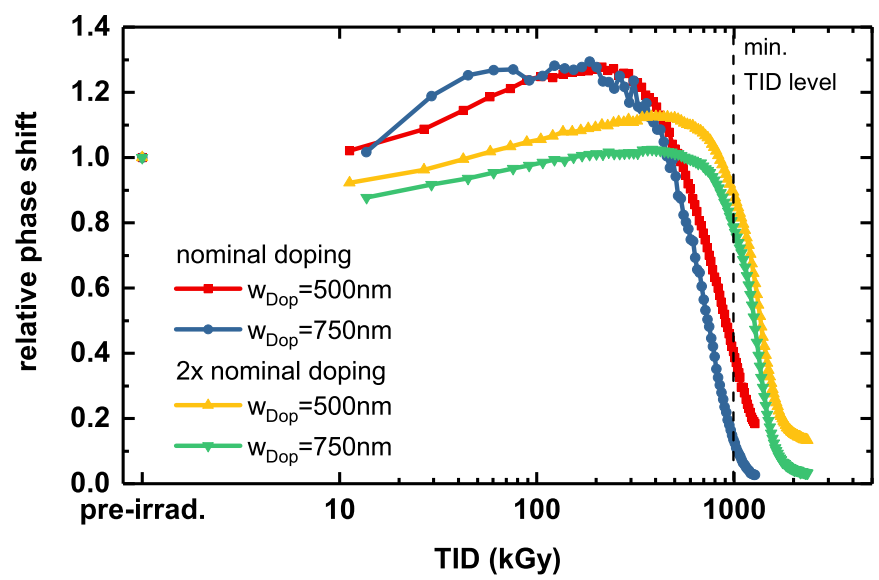

(b)

Fig. 5. Relative phase shift change at $-2 \mathrm{~V}$ as a function of TID obtained during the continuous irradiation test at room temperature where the MZMs were biased at $-3 \mathrm{~V}$ and measured during irradiation. (a) Deep etch. (b) Shallow etch.

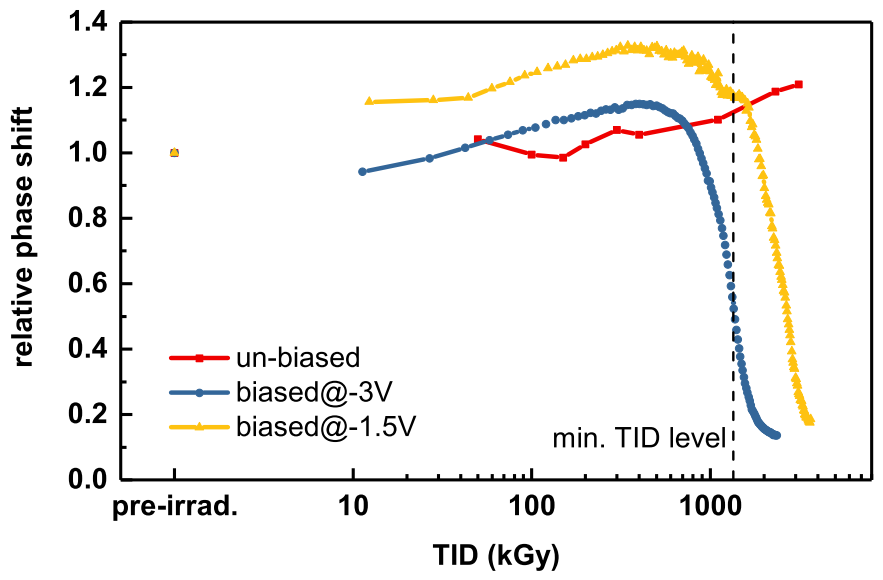

Fig. 6. Phase shifts of shallow etch MZMs with $2 \times$ nominal doping $\left(w_{\text {Dop }}=500 \mathrm{~nm}\right)$ measured during irradiation tests with different bias conditions. The phase shifts were measured at $-2 \mathrm{~V}$.

of the preirradiation value until $2715 \mathrm{kGy} ; 17 \%$ phase shifts were left at a TID level of $3614 \mathrm{kGy}$. Thus, similar to the behavior of CMOS transistors, MZMs that were biased at

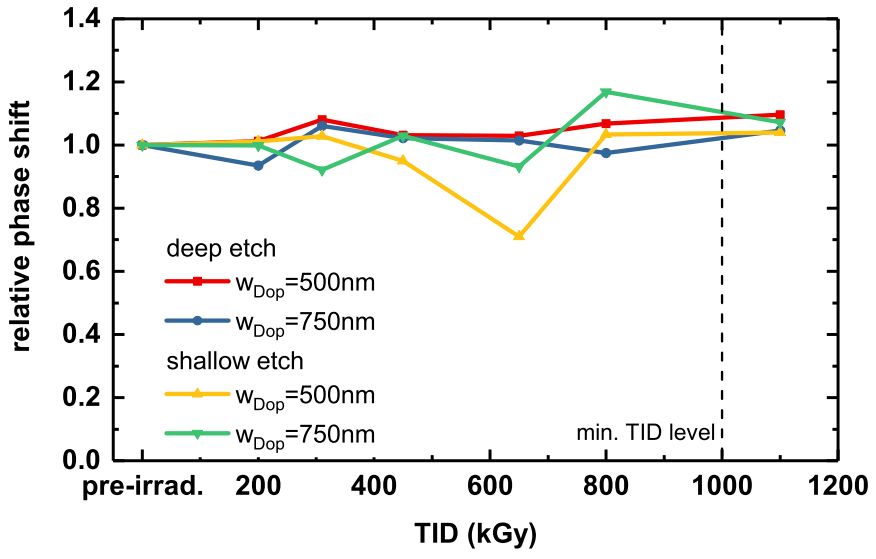

Fig. 7. Relative phase shift change at $-2 \mathrm{~V}$ as a function of TID obtained during the stepwise irradiation test of MZMs with nominal doping at $-30^{\circ} \mathrm{C}$.

larger reverse voltages during irradiation degraded faster than samples biased at lower voltages.

\section{Stepwise Irradiation of MZMs at $-30{ }^{\circ} \mathrm{C}$}

Fig. 7 shows that there was no critical phase shift degradation of neither deep etch nor shallow etch MZMs with nominal doping when they were exposed to ionizing radiation at $-30{ }^{\circ} \mathrm{C}$. The ups and downs in the two graphs are attributed to the fact that a different die was used for each tested TID level. Consequently, one cannot expect a perfect straight line even if the results were normalized to their respective preirradiation values. Additionally, a phase shift enhancement as it was measured during the other two tests is most likely superimposed to this effect. Except for the lower outlier at $650 \mathrm{kGy}$ and the upper outlier at $800 \mathrm{kGy}$, the phase shift of MZMs with both etch depths varied less than $10 \%$ up to a TID of 1.1 MGy. The radiation hardness of deep etch MZMs is thus a factor of at least $11 \times$ better compared with the results collected during the stepwise irradiation at room temperature.

\section{Continuous Irradiation of PDs at Room Temperature}

The continuous irradiation of $\mathrm{GeSi}$ building-block PDs with X-rays showed that these devices were not significantly affected by ionizing radiation up to a TID of 3.6 MGy (Fig. 8). The dark-currents doubled to about $20 \mathrm{nA}$ during irradiation but nonetheless remained low. The photo-currents barely changed during the irradiation. The difference of four orders of magnitude between dark- and photo-current demonstrates that these PDs would have been able to reliably convert an optical data stream back to the electrical domain throughout the irradiation.

\section{DISCUSSION}

Ionizing radiation impinging on SiPh MZMs can lead to the buildup of traps along the silicon/oxide interface [13], [14], the creation and trapping of holes in the oxide [15], [16], and hydrogen-induced passivation of dopants in silicon [17], [18]. Simulations were used to reproduce the observed phase shift enhancement and degradation of SiPh MZMs and understand 


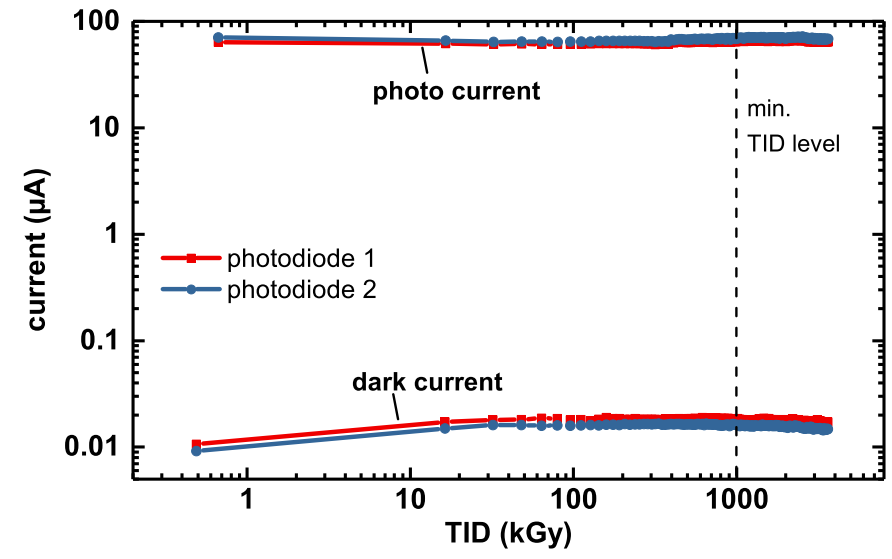

Fig. 8. Dark- and photo-current of building-block PDs as a function of TID obtained during the continuous irradiation test at room temperature where the PDs were biased at $-1 \mathrm{~V}$. Both currents were measured at $-1 \mathrm{~V}$.

the underlying damage mechanisms based on a deep etch MZM with nominal doping. For this purpose, TCAD simulations in Synopsys Sentaurus [19] were performed to calculate the carrier densities in the $p-n$ junctions of the MZMs at the tested bias voltages. These carrier densities were then converted with the Soref-Benett equations [20] into a grid of complex refractive indices. This grid was then imported into PhoeniX OptoDesigner [21] where the effective refractive index $n_{\text {eff }}$ of the fundamental mode in the waveguide for each voltage was found. The phase shift $\Delta \phi$ per unit length for a voltage-induced change in the effective refractive index $\Delta n_{\text {eff }}$ was then calculated with

$$
\Delta \phi=\frac{2 \pi \Delta n_{\mathrm{eff}}}{\lambda}
$$

with $\lambda$ being the wavelength of light.

The simulation model that was used to account for the radiation-induced damage was based on the model published in [8] (the parameter names in the following are the same as used for the published model) and implemented with the Physical Model Interface in Sentaurus. The model accounts for the buildup of interface traps and the trapping of holes in the oxide as a function of TID. Hydrogenation and the transport of radiation-generated holes through the oxide are not included in the model.

Compared with the published model, two modifications were made for this analysis. The electric-field-dependent trapping cross section $\sigma_{\mathrm{ot}}(E)$ was calculated with [22]

$$
\sigma_{\mathrm{ot}}(E)=\frac{\sigma_{0}}{1+1.9 \cdot 10^{-4}|E|^{0.55}}
$$

where $\sigma_{0}$ is the default trapping cross section and $E$ is the magnitude of the electric field. Furthermore, the maximum density of deep traps $N_{\mathrm{HT}}$ in the buried oxide (BOX) is assumed to be lower than that in the top oxide (TOX) cladding because the BOX is typically of higher quality than the faster grown TOX. Therefore, the density of deep traps in the BOX is scaled by a factor $\beta<1$ to that of the TOX.

Given the simplified model, the simulated phase shift of unbiased deep etch MZMs at room temperature agrees well with the measurements (Fig. 9). The simulation parameters

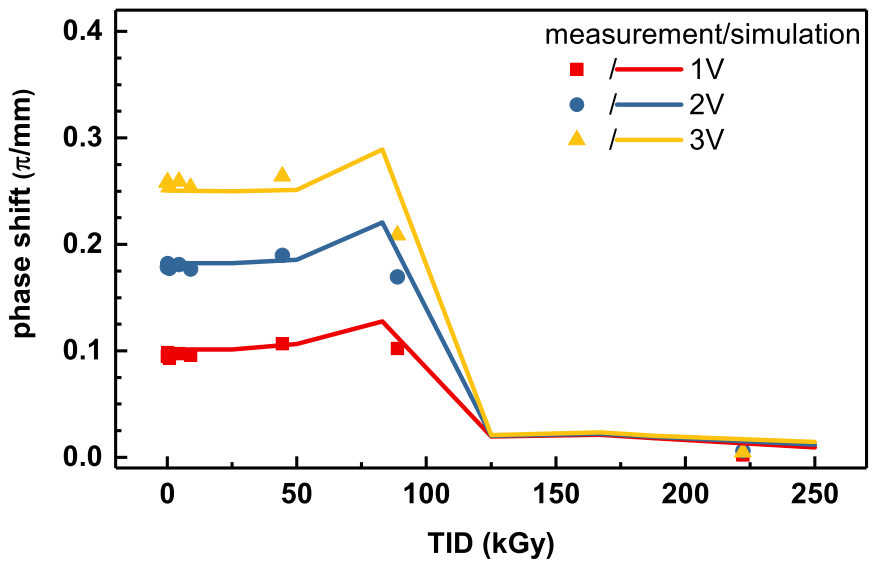

Fig. 9. Comparison of simulated and measured phase shifts as a function of TID for unbiased deep etch MZMs with nominal doping irradiated at room temperature.

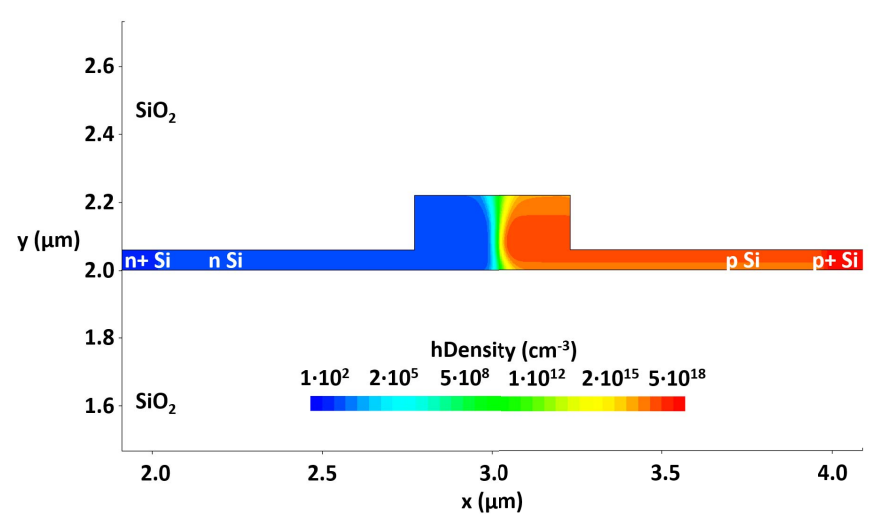

(a)

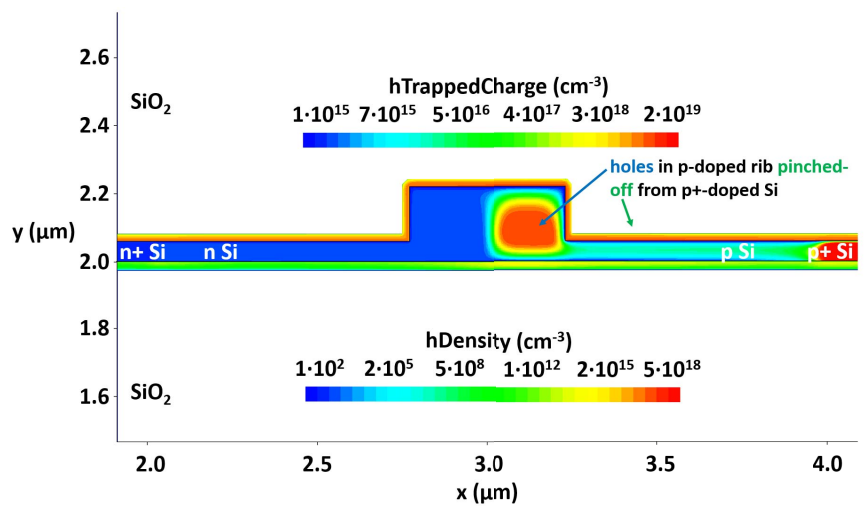

(b)

Fig. 10. Free hole density in the phase shifter (a) before irradiation and (b) after $250 \mathrm{kGy}$ for a deep etch MZM with nominal doping and without bias during irradiation. The density of trapped holes in $\mathrm{SiO}_{2}$ is overlaid in the bottom plot.

used to obtain these results were $\sigma_{0}=6.5 \cdot 10^{-14} \mathrm{~cm}^{2}$, $\Delta X=20 \mathrm{~nm}, \beta=0.3, a_{\mathrm{it}}=5 \cdot 10^{4}\left(\mathrm{rad} \cdot \mathrm{cm}^{2}\right)^{-1}$ for hole interface traps, $a_{\mathrm{it}}=5 \cdot 10^{3}\left(\mathrm{rad} \cdot \mathrm{cm}^{2}\right)^{-1}$ for electron interface traps, $b_{\mathrm{it}}=1$, and $N_{\mathrm{HT}}=5 \cdot 10^{19} \mathrm{~cm}^{-3}$.

Comparing the simulated distributions of free holes in the phase shifter before and after irradiation shows that a pinch-off in the p-doped slab area leads to the device failure after exposure to ionizing radiation (Fig. 10). This pinch-off 


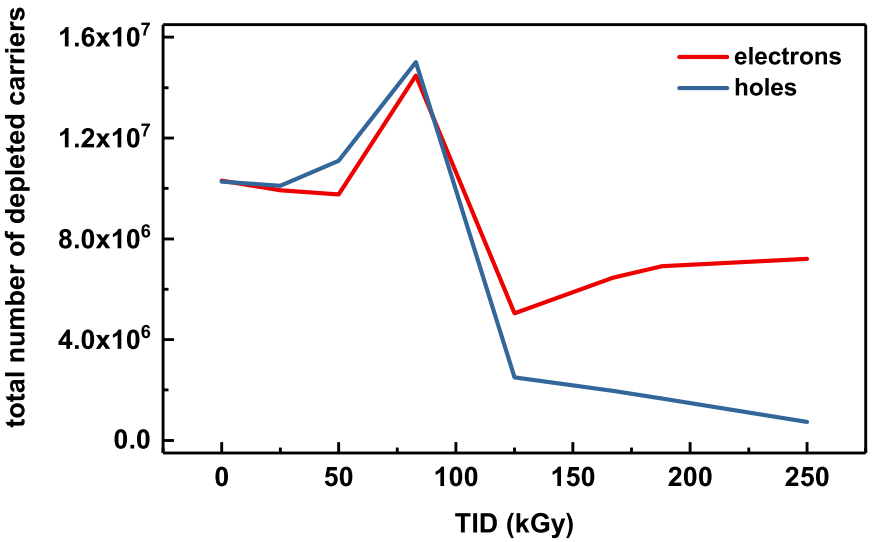

Fig. 11. Total number of depleted carriers at $-3 \mathrm{~V}$ as a function of TID for a deep etch MZM with nominal doping.

is caused by the radiation-induced trapped positive charges in $\mathrm{SiO}_{2}$ and the interface traps along the $\mathrm{Si} / \mathrm{SiO}_{2}$-interface. These defects push the free holes in the p-doped silicon away until holes in the waveguide rib eventually become pinched-off from the $\mathrm{p}+$ doped region that connects to the electrode. Therefore, carriers can no longer be depleted from the p-n junction when a voltage is applied. The phase shift consequently vanishes. This also explains why MZMs with a higher doping concentration or a shallow instead of a deep etch waveguide withstood higher TID levels. In either case, there are more free holes in the p-doped slab. Consequently, a higher concentration of defects, caused by higher TID levels, is required to push the holes away and create a pinch-off.

Integrating over the electron and hole density in the doped $\mathrm{n}$ - and p-silicon and multiplying the integral with the length $L$ of the MZM's phase shifter emphasize this effect. About $10 \times$ fewer holes and only $50 \%$ of electrons are depleted from the phase shifter after $250 \mathrm{kGy}$ when compared with a preirradiation device (Fig. 11).

Fig. 11 also reveals why there is a phase shift enhancement before the phase shift drops. Compared with the preirradiation device, approximately $40 \%$ more electrons and holes are depleted from the p-n junction at $83 \mathrm{kGy}$. The increased number of depleted carriers directly translates to a larger change in the effective refractive index and so in the phase shift. The reason for the increase is two inversion layers in the p-doped silicon slab along the interface that emerge as a result of the trapping of holes in the oxide (Fig. 12). These inversion layers are depleted under reverse bias and the phase shift enhances. Similarly to using a longer phase shifter or applying a larger modulation voltage to create a phase shift as close to $\pi$ as possible, such a phase shift enhancement would increase the difference between the on- and off-state optical power levels of MZMs. Consequently, the quality of the transmitted optical data signals would be improved.

The currently implemented damage model does not include the transport of radiation-generated holes in the oxide. It is hence not possible to simulate the accelerated phase shift degradation when the MZMs are reverse biased during irradiation or the delayed degradation at $-30^{\circ} \mathrm{C}$. We believe that the

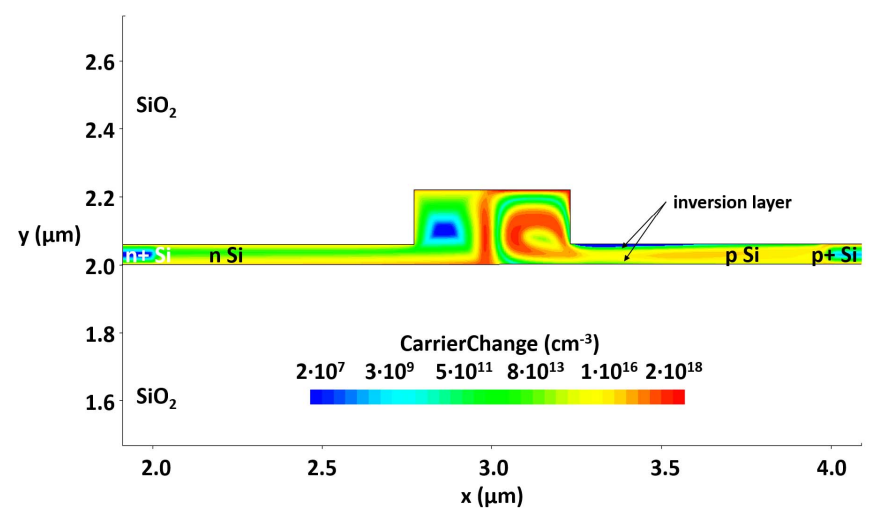

Fig. 12. Sum of depleted electrons and holes at $-3 \mathrm{~V}$ in a deep etch MZM with nominal doping after $83 \mathrm{kGy}$. The inversion layers in silicon along the interface emerge as a result of the trapped positive charge in the oxide.

accelerated degradation under reverse bias has two reasons. First, the yield $Y$ of electron-hole pairs that escape prompt recombination, given by [23]

$$
Y(E)=\left(\frac{E+0.1 \mathrm{~V} / \mathrm{cm}}{E+1.35 \mathrm{MV} / \mathrm{m}}\right)^{0.9}
$$

for X-rays, increases with the electric field strength. As the electric field becomes stronger when the MZMs are reverse biased, the fraction of holes that escape the prompt recombination is larger. The resulting increased number of holes can fill up the deeps traps in the oxide more rapidly and the pinch-off can occur at lower TID levels. Furthermore, the hole velocities in the oxide are faster when the holes travel in stronger electric fields. Thus, they can reach the interface more quickly and either become trapped or induce interface traps.

The delayed degradation at a lower temperature can most likely be attributed to a reduced hole mobility. The hole mobility in $\mathrm{SiO}_{2}$ is about two orders of magnitude lower at $-30{ }^{\circ} \mathrm{C}$ when compared with room temperature [24]. Radiation-generated holes consequently move significantly slower through the oxide and it thus takes longer for them to reach the interface and induce a pinch-off. As a result, the MZMs withstand the radiation longer.

The transport of holes through the oxide and hydrogenation should be implemented in the radiation-damage model to further improve the simulation results and be able to simulate the irradiation at different temperatures and biases. Hydrogenation would be particularly relevant to simulate the irradiation up to higher TID levels as the passivation of dopant then becomes more pronounced [25].

\section{CONClusion}

New optical TRx with higher radiation hardness are required to read out the particle sensors in the innermost regions of future HEP experiments. $\mathrm{SiPh}$ is regarded as a promising technology to address this challenge. In this paper, we presented results from a neutron irradiation test of SiPh MZMs based on a conventional design and different X-ray irradiation campaigns with $\mathrm{SiPh}$ MZMs having varied design parameters 
and building-block PDs. The neutron irradiation test confirmed that the phase shift of SiPh MZMs is not affected up to a $20-\mathrm{MeV}$ neutron fluence of $3 \cdot 10^{16} \mathrm{n} / \mathrm{cm}^{2}$. Furthermore, our $\mathrm{X}$-ray tests showed that reducing the etch depth of the optical waveguide in the $\mathrm{SiPh} \mathrm{MZM}$ and increasing the doping concentrations of the $\mathrm{p}-\mathrm{n}$ junctions improved their resistance against ionizing radiation. In contrast, MZMs degraded faster when they were reverse-biased during irradiation. This accelerated degradation becomes faster for larger reverse biases. Nonetheless, shallow etch MZMs with $2 \times$ nominal doping biased at $-3 \mathrm{~V}$ withstood TID levels of more than 1.3 MGy at room temperature without significant phase shift degradation. This would be enough for a deployment in the innermost regions of future HEP experiments. Even higher TID levels could most likely be reached if $\mathrm{SiPh}$ MZMs were irradiated at lower temperatures. At $-30{ }^{\circ} \mathrm{C}$, unbiased MZMs with nominal doping did not show any degradation in their phase shift up to 1.1 MGy. This is an important finding for radiation-hard optical links from a system perspective. Deep etch MZMs exhibit lower optical losses while at the same time featuring a better phase modulation efficiency compared with shallow etch MZMs. Thus, the minimum required performance for radiation-hard optical links based on deep etch MZMs could be achieved with lower power lasers and with a reduced voltage drive signal compared with optical links based on shallow etch MZMs [26]. The dark- and photo-currents of GeSi PDs were not significantly affected by ionizing radiation up to $3.6 \mathrm{MGy}$.

The phase shift degradation of deep etch MZMs with nominal doping was qualitatively simulated. It could be attributed to a pinch-off of free holes in the waveguide rib from the electrode. Therefore, carriers can no longer be depleted from the phase shifter when a voltage is applied.

As next steps, MZMs should be reverse biased during an irradiation at low temperature to quantify the interplay of the accelerated and delayed degradation due to biasing and reduced temperatures, respectively. A neutron irradiation test of GeSi PDs should also be carried out to determine whether PDs can withstand a neutron fluence as high as MZMs.

\section{REFERENCES}

[1] P. Baldwin and M. Gaillard. (2016). CERN's IT gears up to face the challenges of LHC Run 2. [Online]. Available: http://cerncourier. com/cws/article/cern/65031

[2] J. Troska et al., "Radiation damage studies of lasers and photodiodes for use in multi-Gb/s optical data links," IEEE Trans. Nucl. Sci., vol. 58, no. 6, pp. 3103-3110, Dec. 2011.

[3] A. Candelori, "Radiation-hard detectors for very high luminosity colliders," Nucl. Instrum. Methods Phys. Res. A, Accel. Spectrom. Detect. Assoc. Equip., vol. 560, no. 1, pp. 103-107, May 2006.

[4] G. Casse et al., "Thin silicon detectors for tracking in high radiation environments," in Proc. IEEE Nucl. Sci. Symp. Med. Imag. Conf. Rec. (NSS/MIC), Nov. 2012, pp. 1661-1663.
[5] D. Marris-Morini et al., "A $40 \mathrm{Gbit} / \mathrm{s}$ optical link on a 300-mm silicon platform," Opt. Exp., vol. 22, no. 6, pp. 6674-6679, Mar. 2014.

[6] S. S. El Nasr-Storey et al., "Effect of radiation on a Mach-Zehnder interferometer silicon modulator for HL-LHC data transmission applications," IEEE Trans. Nucl. Sci., vol. 62, no. 1, pp. 329-335, Feb. 2015.

[7] M. Huhtinen, "Simulation of non-ionising energy loss and defect formation in silicon," Nucl. Instrum. Methods Phys. Res. A, Accel. Spectrom. Detect. Assoc. Equip., vol. 491, no. 1, pp. 194-215, 2002.

[8] S. S. El Nasr-Storey et al., "Modeling TID effects in Mach-Zehnder interferometer silicon modulator for HL-LHC data transmission applications," IEEE Trans. Nucl. Sci., vol. 62, no. 6, pp. 2971-2978, Dec. 2015.

[9] M. Zeiler et al., "Design of Si-photonic structures to evaluate their radiation hardness dependence on design parameters," J. Instrum., vol. 11, no. 1, p. C01040, Jan. 2016.

[10] J. Svarny, "Analysis of quadrature bias-point drift of Mach-Zehnder electro-optic modulator," in Proc. IEEE 12th Biennial Baltic Electron. Conf., Tallinn, Estonia, Oct. 2010, pp. 231-234.

[11] imec-ePIXfab SiPhotonics: ISIPP50G. Accesssed: May 10, 2017. [Online]. Available: http://www.europractice-ic.com/SiPhotonics_ technology_imec_ISIPP25G.php

[12] ePIXfab. Accesssed: May 10, 2017. [Online]. Available: http://www. epixfab.eu/

[13] J. Zhang, E. Fretwurst, R. Klanner, I. Pintilie, J. Schwandt, and M. Turcato, "Investigation of X-ray induced radiation damage at the $\mathrm{Si}_{-} \mathrm{SiO}_{2}$ interface of silicon sensors for the European XFEL," J. Instrum., vol. 7, no. 12, p. C12012, Dec. 2012.

[14] H. J. Barnaby, B. Vermeire, and M. J. Campola, "Improved model for increased surface recombination current in irradiated bipolar junction transistors," IEEE Trans. Nucl. Sci., vol. 62, no. 4, pp. 1658-1664, Aug. 2015.

[15] D. M. Fleetwood and J. H. Scofield, "Evidence that similar point defects cause 1/f noise and radiation-induced-hole trapping in metal-oxidesemiconductor transistors," Phys. Rev. Lett., vol. 64, no. 5, pp. 579-582, Jan. 1990.

[16] H. J. Barnaby, "Total-ionizing-dose effects in modern CMOS technologies," IEEE Trans. Nucl. Sci., vol. 53, no. 6, pp. 3103-3121, Dec. 2006.

[17] S. C. Witczak, R. C. Lacoe, M. R. Shaneyfelt, D. C. Mayer, J. R. Schwank, and P. S. Winokur, "Implications of radiation-induced dopant deactivation for npn bipolar junction transistors," IEEE Trans. Nucl. Sci., vol. 47, no. 6, pp. 2281-2288, Dec. 2000.

[18] K. Akarvardar, R. D. Schrimpf, D. M. Fleetwood, S. Cristoloveanu, P. Gentil, and B. J. Blalock, "Evidence of radiation-induced dopant neutralization in partially-depleted SOI NMOSFETs," IEEE Trans. Nucl. Sci., vol. 54, no. 6, pp. 1920-1924, Dec. 2007.

[19] Synopsys Sentaurus Device. Accesssed: May 10, 2017. [Online]. Available: https://www.synopsys.com/silicon/tcad/device-simulation/ sentaurus-device.html

[20] R. A. Soref and B. R. Bennett, "Electrooptical effects in silicon," IEEE J. Quantum Electron., vol. 23, no. 1, pp. 123-129, Jan. 1987.

[21] PhoeniX OptoDesigner 5. Accesssed: May 10, 2017. [Online]. Available: http://www.phoenixbv.com/index.php

[22] P. Paillet, J. L. Touron, J. L. Leray, C. Cirba, and A. Michez, "Simulation of multi-level radiation-induced charge trapping and thermally activated phenomena in $\mathrm{SiO}_{2}$," IEEE Trans. Nucl. Sci., vol. 45, no. 3, pp. 1379-1384, Jun. 1998.

[23] P. Fernández-Martínez, I. Cortés, S. Hidalgo, D. Flores, and F. R. Palomo, "Simulation of total ionising dose in MOS capacitors," in Proc. 8th Spanish Conf. Electron Devices (CDE), Palma de Mallorca, Spain, 2011, pp. 1-4.

[24] R. C. Hughes, "Hole mobility and transport in thin $\mathrm{SiO}_{2}$ films," Appl. Phys. Lett., vol. 26, no. 8, pp. 436-438, Apr. 1975.

[25] E. da Silva, Jr., E. de Vasconcelos, and V. Freire, "Time evolution of $\mathrm{SiO}_{2} / \mathrm{Si}$ interface defects and dopant passivation in MOS capacitors," Microelectron. Eng., vols. 51-52, pp. 567-574, May 2000.

[26] M. Zeiler et al., "A system-level model for high-speed, radiationhard optical links in HEP experiments based on silicon Mach-Zehnder modulators," J. Instrum., vol. 11, no. 12, p. C12059, Dec. 2016. 